Journal of Computer Science 8 (1): 84-88, 2012

ISSN 1549-3636

(C) 2012 Science Publications

\title{
The Effect of Temperature on the Performance of Uncooled Semiconductor Laser Diode in Optical Network
}

\author{
Mohammad Syuhaimi Ab-Rahman and Nurain Izzati Shuhaimi \\ Department of Electrical, Electronics and Systems, \\ Faculty of Engineering and Built Environment \\ University Kebangsaan Malaysia 43600 UKM Bangi, \\ Selangor, Malaysia
}

\begin{abstract}
Problem statement: The characteristics of a laser diode are highly dependent on the temperature of the laser chip. Thus, the effect of temperature on the network performance of uncooled semiconductor laser diode are studied by simulating its equivalent electrical circuit, developed from the rate equations that governing optical components directly into an electrical simulation framework. Approach: The simulations are carried out using circuit analysis program named OptiSPICE and Opti System. On this simulation study, the analysis is done based on three parameters in order to determine the quality of received signal which are; center wavelength, bandwidth and output power. Results: As the temperature variance is fed to the laser diode through an external thermal noise, the result will shows that the ambient temperature of laser diode is varied with time. In this case, the performance of laser diode will decreases as the operating temperature increases or, conversely the performance increases as the operating temperature decreases. Conclusion: The circuit design must be simulated using a SPICE engine that incorporates both electrical and optical models, which is Opti SPICE software. It cannot be simulated using traditional software packages because they do not provide sufficient coupling between electrical and optical devices.
\end{abstract}

Key words: Semiconductor laser diode, temperature effect, center wavelength, output power, provide sufficient, coupling between, software packages, circuit design, incorporates both, optical devices, stimulated emission

\section{INTRODUCTION}

A laser is a device that emits light through a process called stimulated emission. The term laser is an acronym for Light Amplification by Stimulated Emission of Radiation. Laser light is usually spatially coherent, which means that the light either is emitted in a narrow, low-divergence beam, or can be converted into one with the help of optical components such as lenses. Typically, lasers are thought of as emitting light with a narrow wavelength spectrum. This is not true of all lasers, however: some emit light with a broad spectrum, while others emit light at multiple distinct wavelengths simultaneously (Silfvast, 2004).

Today, communication tools such as mobile phone and internet have been widely used and supported our daily lives. Many of those systems are based on broadband optical fiber communication systems because of their large and high-speed transmission capability. In those optical fiber transmission systems, key components are optical sources and optical receivers, especially Semiconductor Laser Diode (SLD). SLD lasing at 1.3 and $1.55 \mu \mathrm{m}$-band are mainly used as the optical sources and their reliability are very important for maintaining their performance in the systems (Ab-Rahman and Hassan, 2010a).

In this simulation study, focus is on SLD. The improvements in laser manufacture today allow operating uncooled direct modulated SLDs over abroad temperature range (Ab-Rahman et al., 2010b). Uncooled SLD are simply wide temperature of operation range, where it is designed to be wider than the span of the environmental operating conditions of communications equipment in which they are used. Uncooled SLD enable low-cost packaging by eliminating many items such as cooler, the thermistor which indicates SLD temperature at any time and the

Corresponding Author: Mohammad Syuhaimi Ab-Rahman, Department of Electrical, Electronics and Systems, Engineering and Built Environment University Kebangsaan Malaysia 43600 UKM Bangi,Selangor, Malaysia 
thermal control loop (Ab-Rahman and Hassan, 2010c). Because a strong cost reduction in optical systems is achieved when eliminating costly laser temperature control, the uncoiled SLDs have been considered as a key technology for future optical network systems (AbRahman et al., 2011). When considering of uncooled SLD in high speed optical communication system, it is high of importance to keep the temperature variation as optimum as possible. It is because; the changes in temperature will translate into changes in wavelength and optical power of SLD. It is known that SLD wavelengths are inherently unstable, where for every 3 degrees' change; the wavelength of the SLD can change nearly $1 \mathrm{~nm}$. If operated in constant current mode, diode output power tends to decrease as temperature increases and diode power can exceed its maximum rating with a decrease in temperature Laser diode principles and diode configurations. For this reason, choosing the constant power control mode of operation is recommended. If not, the temperature of operation will affect seriously the characteristics of SLD.

In laser operation, the thin area of an energy pumping, when the rapid transition from a spontaneous emission mode to a stimulated emission mode occurs, is called the threshold. A SLD is related with a term of threshold density of a driving current or threshold current, when the laser diode operating mode is changed from incoherent to laser. Increasing temperature introduces decreasing of the features of SLD. While the temperature increases, the ratio between the electrical power and the optical power decreases. The temperature also depends on the emitted wavelength or the device operating life. It is important to discuss on an examination of performance of SLD on a temperature variation because by theoretical study, laser thresholds are depends on temperature.

\section{MATERIALS AND METHODS}

The main interface is the OptiSpice schematic, where it is one of among Optiwave design software for photonic. OptiSPICE is circuit design software for analysis of integrated circuits including interactions of optical and electronic components and it is a fullyintegrated solution for parameter extraction, schematic capture, circuit simulation and waveform analysis. The process to design a circuit of uncooled SLD by presence of temperature variation is start by using OptiSPICE schematic framework, where it is illustrated in Fig. 1. The circuit design in OptiSPICE schematic is then visualized in Opti System Graphical User Interface (GUI) to get the simulation results, as shown in Fig. 2. In Opti spice schematic framework design, the initial DC current is kept at $0.078 \mathrm{~A}$ and only the wavelength is changed, where three different wavelengths have been used to analyse the laser performance, i.e., 1550, 1490 and 1450

$\mu \mathrm{m}$.

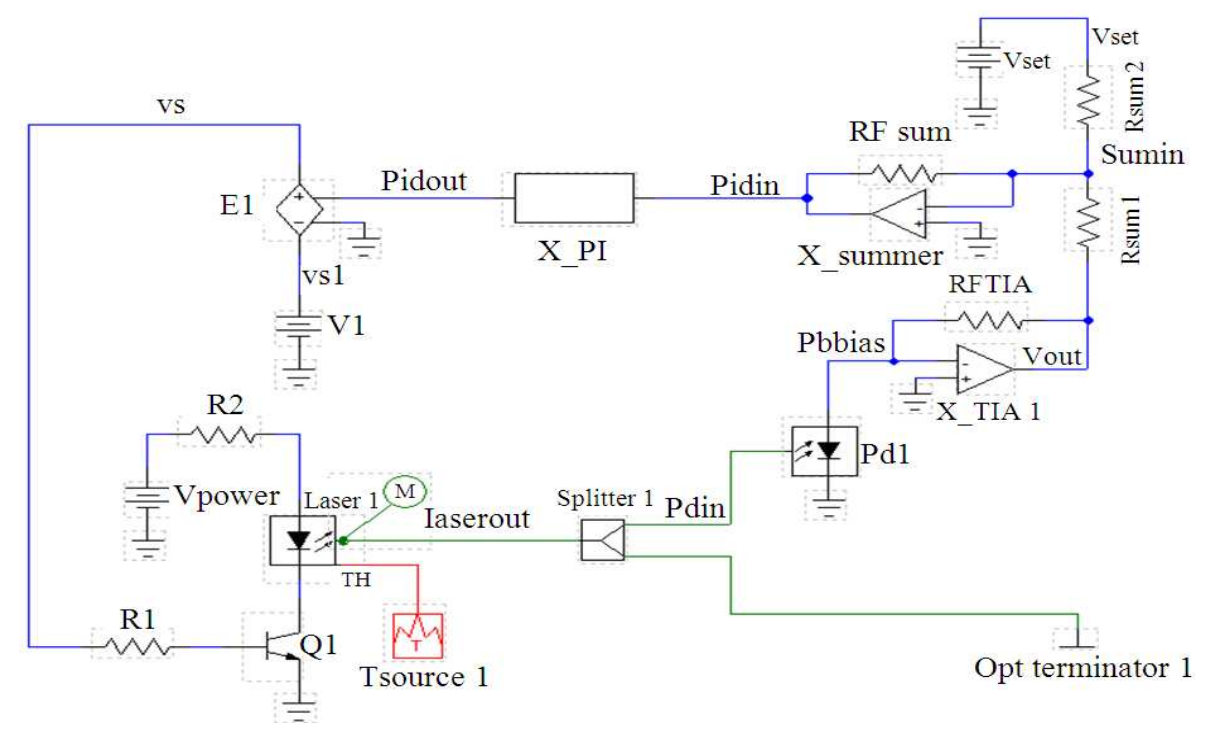

Fig. 1: Point-to-point configuration of SLD design 


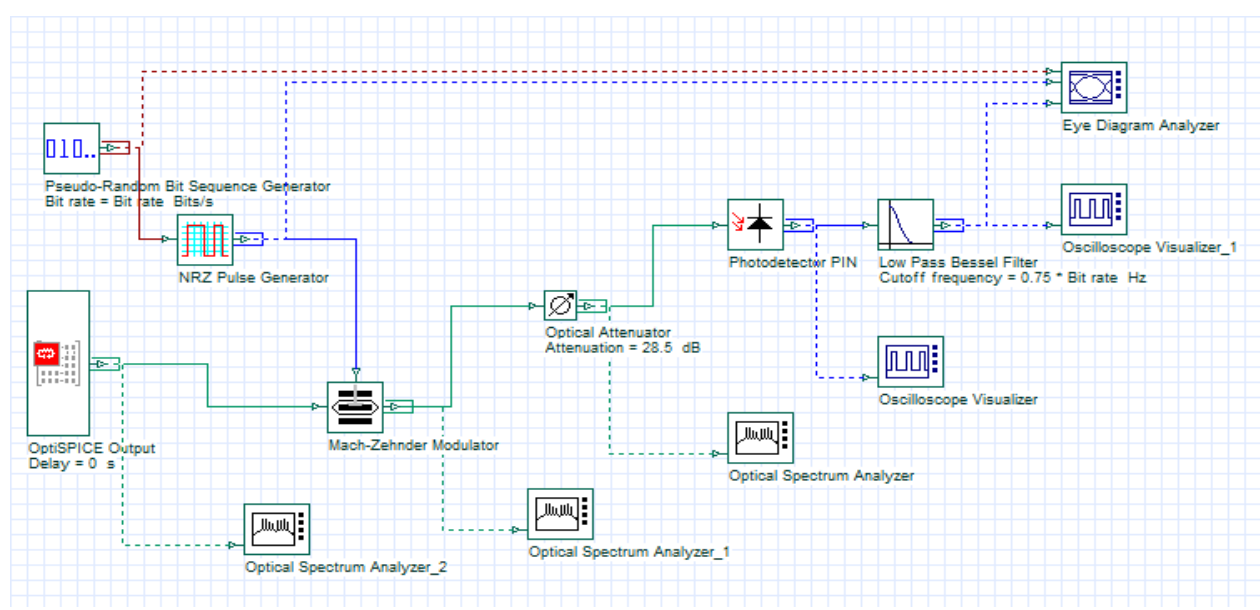

Fig. 2: Connection of components

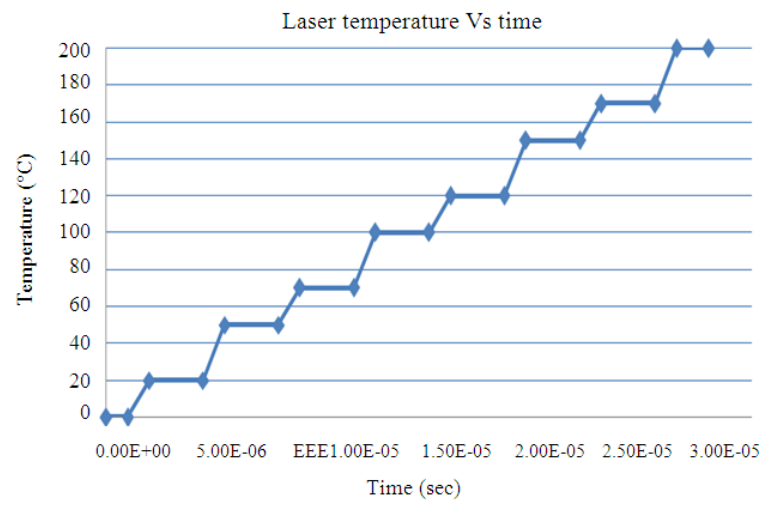

Fig. 3: Laser temperature

Table 1: Summary of temperature description by thermal source

\begin{tabular}{ll}
\hline DC value (C) & Time-temperature values (s, C) \\
\hline 0 & $\{00\}\{1 \mathrm{e}-60\}$ \\
20 & $\{00\}\{1 \mathrm{e}-60\}\{2 \mathrm{e}-620\}\{4.5 \mathrm{e}-620\}$ \\
50 & $\{00\}\{1 \mathrm{e}-60\}\{2 \mathrm{e}-620\}\{4.5 \mathrm{e}-620\}$ \\
70 & $\{5.5 \mathrm{e}-650\}\{8 \mathrm{e}-650\}$ \\
& $\{00\}\{1 \mathrm{e}-60\}\{2 \mathrm{e}-620\}\{4.5 \mathrm{e}-620\}$ \\
& $\{5.5 \mathrm{e}-650\}\{8 \mathrm{e}-650\}\{9 \mathrm{e}-670\}\{11.5 \mathrm{e}-670\}$ \\
& $00\}\{1 \mathrm{e}-60\}\{2 \mathrm{e}-620\}\{4.5 \mathrm{e}-620\}$ \\
& $5.5 \mathrm{e}-650\}\{8 \mathrm{e}-650\}\{9 \mathrm{e}-670\}\{11.5 \mathrm{e}-670\}$ \\
& $\{12.5 \mathrm{e}-6100\}\{15 \mathrm{e}-6100\}$ \\
& $\{00\}\{1 \mathrm{e}-60\}\{2 \mathrm{e}-620\}\{4.5 \mathrm{e}-620\}\{5.5 \mathrm{e}-650\}$ \\
& $\{8 \mathrm{e}-650\}\{9 \mathrm{e}-670\}\{11.5 \mathrm{e}-670\}\{12.5 \mathrm{e}-6100\}$ \\
& $\{15 \mathrm{e}-6100\}\{16 \mathrm{e}-6120\}\{18.5 \mathrm{e}-6120\}$ \\
& $\{00\}\{1 \mathrm{e}-60\}\{2 \mathrm{e}-620\}\{4.5 \mathrm{e}-620\}\{5.5 \mathrm{e}-650\}$ \\
& $\{8 \mathrm{e}-650\}\{9 \mathrm{e}-670\}\{11.5 \mathrm{e}-670\}\{12.5 \mathrm{e}-6100\}$ \\
& $\{15 \mathrm{e}-6100\}\{16 \mathrm{e}-6120\}\{18.5 \mathrm{e}-6120\}$ \\
& $\{19.5 \mathrm{e}-6150\}\{22 \mathrm{e}-6150\}$ \\
& $\{8 \mathrm{e}-650\}\{0\}$ e 50$\}-670\}\{11.5 \mathrm{e}-670\}\{12.5 \mathrm{e}-6100\}$ \\
& $\{15 \mathrm{e}-6100\}\{16 \mathrm{e}-6120\}\{18.5 \mathrm{e}-6120\}\{19.5$ \\
& $\mathrm{e}-6150\}\{22 \mathrm{e}-6150\}\{23 \mathrm{e}-6170\}\{25.5 \mathrm{e}-6170\}$ \\
& $\{00\}\{1 \mathrm{e}-60\}\{2 \mathrm{e}-620\}\{4.5 \mathrm{e}-620\}\{5.5 \mathrm{e}-650\}$ \\
& $\{8 \mathrm{e}-650\}\{9 \mathrm{e}-670\}\{11.5 \mathrm{e}-670\}\{12.5 \mathrm{e}-6100\}$ \\
& $\{15 \mathrm{e}-6100\}\{16 \mathrm{e}-6120\}\{18.5 \mathrm{e}-6120\}$ \\
& $\{19.5 \mathrm{e}-6150\}\{22 \mathrm{e}-6150\}\{23 \mathrm{e}-6170\}$ \\
& $\{25.5 \mathrm{e}-6170\}\{26.5 \mathrm{e}-6200\}\{28 \mathrm{e}-6200\}$ \\
\hline
\end{tabular}

Table 2: Layout parameter's detail in OptiSystem GUI

\begin{tabular}{ll}
\hline Parameter & Value \\
\hline Bit rate & $1 \mathrm{e} 9 \mathrm{bits} \mathrm{sec}^{-1}$ \\
Sequence length & $128 \mathrm{bits}$ \\
Samples per bit & 64 \\
No. of samples & 8192 \\
\hline
\end{tabular}

In this design, ambient temperature of the laser is varied with time as shown in Fig. 3. This temperature variance is fed to the laser through an external thermal source and for each wavelength; the summary of injected temperature is provided in Table 1.

While in OptiSystem GUI, the attenuation at optical attenuator is maintained at $28.5 \mathrm{~dB}$ and layout parameters are changed, as listed in Table 2.

\section{RESULTS}

Figure 4-6 show the simulated result of point-to-point configuration of SLD design for three different wavelengths; 1550,1490 and $1450 \mathrm{~nm}$, which respectively for center wavelength, bandwidth and output power.

By looking at Fig. 4, it shows that the center wavelength of a laser diode is directly proportional to its operating temperature. There is almost linear relationship between temperature and center wavelength. As temperature increases, so does the center wavelength of the laser diode.

Increased bandwidth means more data per unit time but also more impairment through higher power and increased channel counts. From the result in Fig. 5, it shows that the higher the operating temperature of laser diode, the wider the bandwidth will be. In addition; for a smaller operating temperature, the narrow the bandwidth will be so that an excellent performance and outstanding reliability will be provided. 


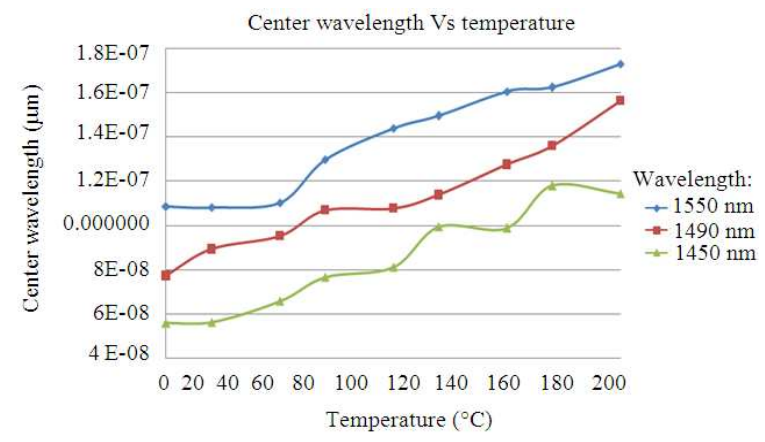

Fig. 4: Effects of temperature on center wavelength

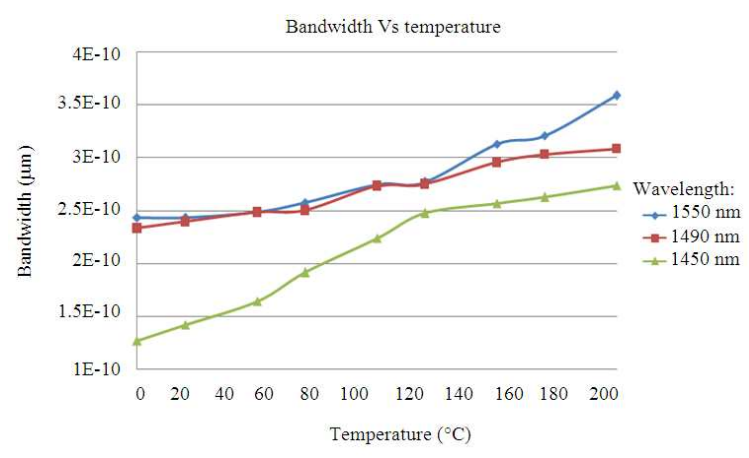

Fig. 5: Effects of temperature on bandwidth

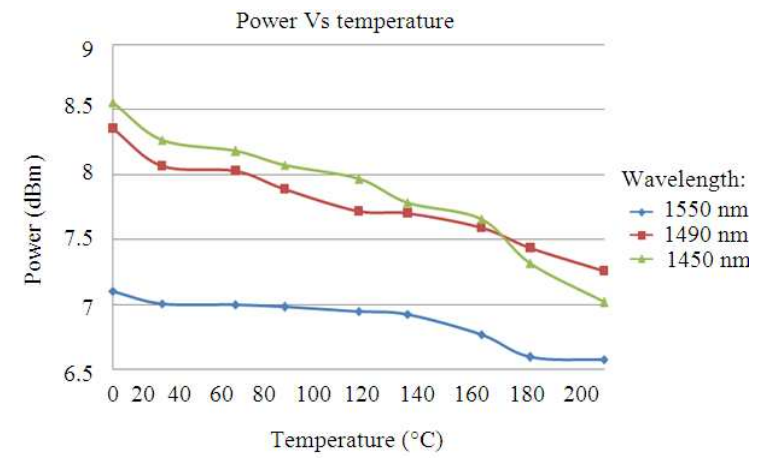

Fig. 6: Effects of temperature on power

The output characteristics of laser diode are strongly dependent on the operating temperature. Figure 6 shows how the output power curve changes with operating temperature for typical laser diodes, where the relationship for the laser diode tested in this simulation is plotted; the decrease in output power corresponds to different wavelengths with increasing operating temperature.

\section{DISCUSSION}

The characteristic that is found from the result is useful in spectroscopy applications, laser diode pumping of solid state lasers and erbium-doped fiber amplifiers, where the wavelength of emission of the laser diode can be accurately temperature-tuned to the specific properties of the material with which it is interacting (Moharban, 2009).

In the other case, for every different wavelength, the bandwidth value is changed relatively to the different value of operating temperature.

Also, from the result found in Fig. 5, it has proven that the bandwidth is directly proportional with the operating temperature of laser diode. Note that laser diode produce light over some natural bandwidth or range of frequencies. A laser's bandwidth of operation is determined primarily by the gain medium that the laser is constructed from and the range of operating temperature that a laser may operate over is known as the gain bandwidth (Zhang et al., 2009). In this system, the gain bandwidth can limit the usable transmission bandwidth and thus the achievable data rate.

From the result in Fig. 6, we found that the temperature affected the intensity. The higher the working temperature, the lower the intensity of the laser radiation hence the power of the laser diode also decreases (Zulkepli and Bidin, 2008). Therefore it is necessary to control the working temperature of laser diode to obtain the most efficient output.

In this study, in order to observe the temperature's effect on laser performance, the three conditions with varying wavelength has been simulated using the OptiSPICE and OptiSystem tools which are effect on center wavelength, bandwidth and output power. However, please take note that temperature can cause references to shift in gain control, bias and threshold detection circuit where it can cause drift in bandwidthdetermining components, thereby increasing signal distortion, intersymbol interference or noise (Jenkins and White, 1976). Temperature also can affect the physical fiber-to-device coupling.

\section{CONCLUSION}

In this simulation study, it was shown the important dependences of laser features on temperature. The temperature can have negative effect on the important parameters and quality of a semiconductor laser diode. There is a need to know the dependency of parameters on the temperature for successful diode construction. It is well known that a laser diode can study at a low constant temperature. In conclusion, the circuit design must be simulated using a SPICE engine that incorporates both electrical and optical models, which is OptiSPICE software. It cannot be simulated using traditional software packages that rely on co-simulation or equivalent circuit models, as they do not provide sufficient coupling between electrical and optical 
devices. The process is continuing until the result on effect of un cooled semiconductor laser diode in presence of temperature variation is achieved.

\section{ACKNOWLEDGEMENT}

The researcher would like to acknowledge University Kebangsaan Malaysia for supporting this research by providing equipment, material and sources of references in accomplishing this research.

\section{REFERENCES}

Ab-Rahman, M.S. and M.R. Hassan, 2010a. Temperature dependence of turn-on time delay of semiconductor laser diode. Theoretical analysis. Opto. Elec. Rev., 18: 458-466. DOI: 10.2478/s11772-010-0015-x

Ab-Rahman, M.S. and M.R. Hassan, 2010b. Theoretical analysis of the effect of temperature dependence of auger coefficient on the turn-on time delay of uncooled semiconductor laser diode. Optics Commun., 283: 2378-2384. DOI: 10.1016/j.optcom.2010.01.071

Ab-Rahman, M.S. and M.R. Hassan, 2010c. Theory of cut-off temperature of operation of uncooled semiconductor laser diode. Cambridge University Press.
Ab-Rahman, M.S. and M.R. Hassan, 2011. The combined effect of temperature of operation and external optical feedback on the turn-on time delay of semiconductor laser diodes. Opt. Int. J. Light Elec. Optics, 122: 266-272. DOI: 10.1016/j.ijleo.2010.01.002

Jenkins, F.A. and H.E. White, 1976. Fundamentals of Optics: An Introduction for Beginners. 4th Edn. Mcgraw-Hill College, USA., ISBN-10: 0070323305, pp: 746.

Moharban, K.S., 2009. Test and Characterization of Laser Diode. Determination of Principal Parameters. 1st Edn. Newport Corporation, USA., pp: $1-10$.

Silfvast, W.T., 2004. Laser Fundamental. 2nd Edn. Cambridge University Press, New York, pp: 642.

Zulkepli, N. and N. Bidin, 2008. The effect of temperature on high power laser diode. J. Phys. UTM., 3: 84-89.

Zhang, H., Q. Bao, D. Tang, L. Zhao, and K. Loh, 2009. Large energy soliton erbium-doped fiber laser with a graphene-polymer composite mode locker. Appl. Phys. Lett., 95: 1-3. DOI: 10.1063/1.3244206 